COE-RES Discussion Paper Series

Center of Excellence Project

The Normative Evaluation and Social Choice of

Contemporary Economic Systems

Graduate School of Economics and Institute of Economic Research Hitotsubashi University

COE/RES Discussion Paper Series, No.209

June 2007

Continuity and Egalitarianism in the Evaluation of Infinite Utility Streams

Chiaki Hara

(Kyoto University)

Tomoichi Shinotsuka

(University of Tsukuba)

Kotaro Suzumura

(Hitotsubashi University)

Yongsheng $\mathrm{Xu}$

(Georgia State University and Central University of Finance and Economics)

Naka 2-1, Kunitachi, Tokyo 186-8603, Japan

Phone: +81-42-580-9076 Fax: +81-42-580-9102

URL: http://www.econ.hit-u.ac.jp/ coe-res/index.htm

E-mail: coe-res@econ.hit-u.ac.jp 


\title{
Continuity and Egalitarianism in the Evaluation of Infinite Utility Streams*
}

\author{
Chiaki Hara \\ Institute of Economic Research, Kyoto University \\ Tomoichi Shinotsuka \\ Graduate School of Humanities and Social Sciences, University of Tsukuba \\ Kotaro Suzumura \\ Institute of Economic Research, Hitotsubashi University \\ and \\ Yongsheng $\mathrm{Xu}$ \\ Andrew Young School of Policy Studies, Georgia State University \\ and \\ Central University of Finance and Economics
}

June 8, 2007

\author{
Corresponding Author \\ Professor Kotaro Suzumura \\ Institute of Economic Research \\ Hitotsubashi University \\ Naka 2-1, Kunitachi, Tokyo 186-8603, Japan \\ Phone \& Fax: 81-42-580-8353 E-mail: suzumura@ier.hit-u.ac.jp
}

*This paper is a substantially revised version of our foregoing paper, "On the Possibility of Continuous, Paretian and Egalitarian Evaluation of Infinite Utility Streams," which was based on our joint research conducted as a part of the Project on Intergenerational Equity under the auspices of the Grant-in-Aid for Scientific Research from the Ministry of Education, Culture, Sports, Science and Technology of Japan. Thanks are due to Geir Asheim, Kaushik Basu, Walter Bossert, Hajime Hori, Tomoki Inoue, Mitsunori Noguchi and Koichi Tadenuma with whom we had several discussions on this and related issues. We are also grateful to the anonymous referees and the Associate Editor in charge of our earlier paper for their helpful comments and suggestions. Needless to say, no one other than ourselves should be held responsible for any defect of this paper which may still remain. 


\begin{abstract}
There exists a utilitarian tradition à la Sidgwick of treating equal generations equally. Diamond showed that there exists no social evaluation ordering over infinite utility streams in the presence of the Pareto principle, the Sidgwick principle, and continuity. Instead of requiring the Sidgwick principle of procedural fairness, we focus on two principles of distributional egalitarianism along the line of the Pigou-Dalton transfer principle and the Lorenz domination principle, and show that there exists no social evaluation relation satisfying one of these egalitarian principles and the weakened continuity and rationality axioms even in the absence of the Pareto principle.
\end{abstract}

JEL Classification Nos.: D63, D71 


\section{Introduction}

According to Aristotle's Politics, "[e]quality consists in the same treatment of similar persons." ${ }^{1}$ One way to make this abstract equity principle operational is to rephrase it as a requirement of anonymity to the effect that one distribution of transferable benefits among persons should be judged socially equivalent to any interpersonal permutation thereof. As a modern resurgence of Aristotle's equity principle, there is a strong utilitarian tradition of treating otherwise equal generations equally. As Sidgwick (1907, p.414) put it, "the time at which a man exists cannot affect the value of his happiness from a universal point of view." However, a serious doubt was raised by Koopmans (1960) on the sustainability of this principle by showing that the rational, continuous, and stationary evaluation of infinite allocation programs cannot but exhibit a phenomenon which he christened impatience, viz., the preference for advancement along the time axis of an outcome yielding higher utility vis-à-vis another outcome yielding lower utility. $^{2}$ This intriguing thesis was elaborated further by Diamond (1965) into a general impossibility theorem to the effect that there exists no social evaluation ordering over the set of infinite utility streams which satisfies the Pareto principle, the equity principle à la Aristotle and Sidgwick in the form of anonymity, and continuity with respect to the sup topology. His work was followed by, among others, Banerjee and Mitra (2007b), Campbell (1985), Lauwers (1997), Shinotsuka (1998), and Svensson (1980), who examined the sensitivity of Diamond's impossibility theorem on the choice of underlying topology. Basu and Mitra (2003) dealt with the case where social evaluation orderings need not be continuous, but they can be represented by numerical functions. Recent studies by Lauwers (2007) and Zame (2007) are concerned with the conjecture due to Fleurbaey and Michel (2003). According to Lauwers and Zame, the existence of any (complete and transitive) social evaluation ordering that satisfies the Pareto principle and the Aristotle-Sidgwick equity principle must necessarily be based on the axiom of choice. Since they work with different domains of the social evaluation orderings, their arguments are quite different, and their results are non-comparable with each other.

Recollect that the equity principle à la Aristotle and Sidgwick does not embody any preference for egalitarian distribution of utilities among generations. Recently, there have been attempts to introduce egalitarian principles that incorporate preferences for egalitarian distribution of utilities among generations. For example, Asheim and Tungodden (2004) and Bossert, Sprumont and Suzumura (2005) extended Hammond's (1976) equity axiom formulated in the traditional framework of social choice theory to the context of ranking infinite utility streams. Likewise, Fleurbaey and Michel (2001; 2003), Hara, Shinotsuka, Suzumura and Xu (2005) and Sakai (2006) introduced two versions of distributional egalitarianism in the spirit of Atkinson (1970) and Sen (1997), viz., the Pigou-Dalton transfer principle and the Lorenz domination principle.

\footnotetext{
${ }^{1}$ Aristotle, The Politics, edited by S. Everson, Cambridge, UK: Cambridge University Press, 1988, p.176.

${ }^{2}$ See, also, Banerjee and Mitra (2007a) who identified the cardinality and the topological structure of the set of infinite utility streams at which the social evaluation ordering exhibits impatience.
} 
While the Pigou-Dalton transfer principle is concerned with the comparison between two utility streams differing from each other only in two generations, the Lorenz domination principle is concerned with the comparison between two utility streams differing from each other in any finitely many generations. We show that there exists no social evaluation relation satisfying one of these egalitarian principles and the weakened continuity and rationality axioms even in the absence of the Pareto principle.

Apart from this Introduction, the paper consists of four sections. Section 2 introduces our notation, model and continuity axioms. Section 3 introduces the axioms of distributional egalitarianism in the spirit of the Pigou-Dalton transfer principle, on the one hand, and in the spirit of the Lorenz domination principle, on the other hand. It is also in this section that we show the non-existence of a social evaluation relation over infinite utility streams satisfying one of our axioms of distributional egalitarianism and the weakened continuity and rationality axioms even in the absence of the Pareto principle. Throughout section 2 and section 3, we invoke the sup topology in defining the weakened continuity axioms on the social evaluation relation over infinite utility streams. In section 4, we examine the extent to which our impossibility theorems are robust with respect to the choice of alternative topologies once the Pareto principle is imposed on social evaluation relations. Section 5 concludes the paper with a few final remarks on and comparison with the existing literature.

\section{Notation and Model}

Let $\mathbb{R}$ and $\mathbb{N}$ denote the set of all real numbers, and the set of all positive integers, respectively. Let $l^{\infty}$ be the set of all bounded infinite sequences of real numbers. In what follows, the domain $X \subset l^{\infty}$ is the set of all infinite utility streams, viz., $\boldsymbol{x}=\left(x_{1}, x_{2}, \ldots, x_{n}, \ldots\right) \in X$ denotes an infinite sequence of utilities, where $x_{n} \in \mathbb{R}$ denotes the utility of generation $n \in \mathbb{N}$. For all $\boldsymbol{x}=$ $\left(x_{1}, x_{2}, \ldots, x_{n}, \ldots\right), \boldsymbol{y}=\left(y_{1}, y_{2}, \ldots, y_{n}, \ldots\right) \in X, \boldsymbol{x} \geq \boldsymbol{y}$ means that $x_{n} \geq y_{n}$ for all $n \in \mathbb{N} ; \boldsymbol{x}>\boldsymbol{y}$ means that $\boldsymbol{x} \geq \boldsymbol{y}$ and $\boldsymbol{x} \neq \boldsymbol{y} ; \boldsymbol{x} \gg \boldsymbol{y}$ means that $x_{n}>y_{n}$ for all $n \in \mathbb{N}$. Denote by $l_{+}^{\infty}$ the set of all $\boldsymbol{x} \in l^{\infty}$ with $\boldsymbol{x} \geq \mathbf{0}$. We assume that $X \neq \varnothing$ and $X+l_{+}^{\infty} \subseteq X$. The sup distance being invariant under translation, we may moreover assume that $l_{+}^{\infty} \subseteq X$. The sup distance between $\boldsymbol{x}$ and $\boldsymbol{y}$ is defined by

$$
d_{s}(\boldsymbol{x}, \boldsymbol{y})=\sup _{n}\left|x_{n}-y_{n}\right|,
$$

which induces the sup topology on the domain $X$.

Let $R$ be a social evaluation relation on $X$, viz., $\boldsymbol{x} R \boldsymbol{y}$ for any pair $\boldsymbol{x}, \boldsymbol{y} \in$ $X$ means that the infinite utility stream $\boldsymbol{x}$ is judged to be at least as good as another infinite utility stream $\boldsymbol{y}$. $P(R)$ denotes the asymmetric part of $R$, viz., for all $\boldsymbol{x}, \boldsymbol{y} \in X, \boldsymbol{x} P(R) \boldsymbol{y}$ holds if and only if $\boldsymbol{x} R \boldsymbol{y}$ and not $\boldsymbol{y} R \boldsymbol{x}$. $I(R)$ denotes the symmetric part of $R$, viz., for all $\boldsymbol{x}, \boldsymbol{y} \in X, \boldsymbol{x} I(R) \boldsymbol{y}$ holds if and only if $\boldsymbol{x} R \boldsymbol{y}$ and $\boldsymbol{y} R \boldsymbol{x}$.

$R$ is said to be complete if and only if, for all $\boldsymbol{x}, \boldsymbol{y} \in X, \boldsymbol{x} R \boldsymbol{y}$ or $\boldsymbol{y} R \boldsymbol{x}$ holds. $R$ is said to be transitive if and only if, for all $\boldsymbol{x}, \boldsymbol{y}, \boldsymbol{z} \in X, \boldsymbol{x} R \boldsymbol{y}$ and $\boldsymbol{y} R \boldsymbol{z}$ imply $\boldsymbol{x} R \boldsymbol{z}$. $R$ is said to be an ordering if and only if it satisfies completeness as well 
as transitivity. Unlike most of the preceding work along the line of Koopmans (1960) and Diamond (1965), where the social evaluation relation is assumed to be an ordering on $X$, this paper invokes much weaker rationality properties of quasi-transitivity and acyclicity in Section 3, which are defined as follows. $R$ is said to be quasi-transitive if and only if $P(R)$ is transitive. For any $t \in \mathbb{N}$, a finite subset $\left\{\boldsymbol{x}^{1}, \boldsymbol{x}^{2}, \ldots, \boldsymbol{x}^{t}\right\}$ of $X$ is called a $P(R)$-cycle of order $t$ if and only if $\boldsymbol{x}^{1} P(R) \boldsymbol{x}^{2}, \boldsymbol{x}^{2} P(R) \boldsymbol{x}^{3}, \ldots, \boldsymbol{x}^{t} P(R) \boldsymbol{x}^{1}$ hold. $R$ is said to be acyclic if and only if there exists no $P(R)$-cycle of any order $t$, where $2<t<+\infty$. It is clear that the transitivity of $R$ implies the quasi-transitive thereof, which in turn implies the acyclicity of $R$. In both cases, the converse implications do not hold in general. Lastly, we say that $R$ is negatively transitive if, for all $\boldsymbol{x}, \boldsymbol{y}, \boldsymbol{z} \in X$, [not $\boldsymbol{x} P(R) \boldsymbol{y}$, and not $\boldsymbol{y} P(R) \boldsymbol{z}$ ] then not $\boldsymbol{x} P(R) \boldsymbol{z}$. It is easy to check that negative transitivity of $R$ is equivalent to transitivity thereof in the presence of completeness.

For any fixed $\boldsymbol{x} \in X$, define the upper contour set of $R$ at $\boldsymbol{x} \in X$ by

$$
U_{R}(\boldsymbol{x})=\{\boldsymbol{y} \in X \mid \boldsymbol{y} R \boldsymbol{x}\},
$$

the lower contour set of $R$ at $\boldsymbol{x} \in X$ by

$$
L_{R}(\boldsymbol{x})=\{\boldsymbol{y} \in X \mid \boldsymbol{x} R \boldsymbol{y}\},
$$

the upper contour set of $P(R)$ at $\boldsymbol{x} \in X$ by

$$
U_{P(R)}(\boldsymbol{x})=\{\boldsymbol{y} \in X \mid \boldsymbol{y} P(R) \boldsymbol{x}\},
$$

and the lower contour set of $P(R)$ at $\boldsymbol{x} \in X$ by

$$
L_{P(R)}(\boldsymbol{x})=\{\boldsymbol{y} \in X \mid \boldsymbol{x} P(R) \boldsymbol{y}\} .
$$

Concerning the continuity requirement on $R$, we will consider the following upper semi-continuity and lower semi-continuity axioms, each of which is weaker than Diamond's full continuity axiom.

R-Upper Semi-Continuity with Respect to the Sup Topology (RUSCs)

For all $\boldsymbol{x} \in X, U_{R}(\boldsymbol{x})$ is closed with respect to the sup topology on $X$.

R-Lower Semi-Continuity with Respect to the Sup Topology (RLSCs)

For all $\boldsymbol{x} \in X, L_{R}(\boldsymbol{x})$ is closed with respect to the sup topology on $X$.

P-Upper Semi-Continuity with Respect to the Sup Topology (PUSCs)

For all $\boldsymbol{x} \in X, L_{P(R)}(\boldsymbol{x})$ is open with respect to the sup topology on $X$.

P-Lower Semi-Continuity with Respect to the Sup Topology (PLSCs)

For all $\boldsymbol{x} \in X, U_{P(R)}(\boldsymbol{x})$ is open with respect to the sup topology on $X$.

If $R$ is complete, then $U_{R}(\boldsymbol{x})=X \backslash L_{P(R)}(\boldsymbol{x})$ and $L_{R}(\boldsymbol{x})=X \backslash U_{P(R)}(\boldsymbol{x})$. Thus, in this case, RUSCs is equivalent to PUSCs, and RLSCs is equivalent to PLSCs. 


\section{Pigou-Dalton Transfer Principle, Lorenz Domination Principle, and Related Axioms of Distributional Egali- tarianism}

In contrast with the equity principle à la Sidgwick and Diamond in the form of anonymity, we consider two axioms which embody alternative forms of preference for egalitarian distribution of utilities among generations.

Our first axiom has been introduced in the literature by, for example, Bossert, Sprumont and Suzumura (2005), and Sakai (2006), which reads as follows:

Pigou-Dalton Transfer Principle (PDT)

For any $\boldsymbol{x}, \boldsymbol{y} \in X$, and any pair of positive integers $i, j \in \mathbb{N}$, if there exists an $\epsilon>0$ such that

$$
y_{i}=x_{i}+\epsilon \leq x_{j}-\epsilon=y_{j}, x_{k}=y_{k} \text { for all } k \in \mathbb{N} \backslash\{i, j\}
$$

holds, then $\boldsymbol{y} P(R) \boldsymbol{x}$ must also hold.

We also introduce an alternative formulation of egalitarian preferences on the set of infinite utility streams in terms of the concept of Lorenz domination. For each $\boldsymbol{x} \in X$ and each $n \in \mathbb{N}$, let ${ }_{1} \boldsymbol{x}_{n}$ be defined by ${ }_{1} \boldsymbol{x}_{n}=\left(x_{1}, x_{2}, \ldots, x_{n}\right)$. For any $n \in \mathbb{N}$, let us say that ${ }_{1} \boldsymbol{x}_{n}$ Lorenz dominates ${ }_{1} \boldsymbol{y}_{n}$ if and only if (i) $\sum_{t=1}^{n} x_{t}=\sum_{t=1}^{n} y_{t}$ and (ii) if $\varphi$ and $\psi$ are permutations on $\{1, \ldots, n\}$ such that $x_{\varphi(1)} \leq \cdots \leq x_{\varphi(n)}$ and $y_{\psi(1)} \leq \cdots \leq x_{\psi(n)}$, then $\sum_{t=1}^{m} x_{\varphi(t)} \geq \sum_{t=1}^{m} y_{\psi(t)}$ for every $m=1, \ldots, n$, with a strict inequality holding for some $m$.

Lorenz Domination Principle (LD)

For any $\boldsymbol{x}, \boldsymbol{y} \in X$, if there exists $n^{*} \in \mathbb{N}$ such that (a) ${ }_{1} \boldsymbol{x}_{n^{*}}$ Lorenz dominates ${ }_{1} \boldsymbol{y}_{n^{*}}$, and (b) $x_{n}=y_{n}$ for all $n \in \mathbb{N}$ such that $n>n^{*}$, then $\boldsymbol{x} P(R) \boldsymbol{y}$ must hold.

Observe that LD implies PDT. With PDT and LD close at hand, we are ready to present the following impossibility results. ${ }^{3}$

\section{Theorem 1}

There exists no acyclic social evaluation relation $R$ satisfying PDT and PUSCs.

\section{Theorem 2}

There exists no social evaluation relation $R$ satisfying LD and PUSCs.

To prove Theorem 1, we first introduce a symbol. For every $\boldsymbol{x}, \boldsymbol{y} \in X$, $\boldsymbol{y} R_{\mathrm{PD}} \boldsymbol{x}$ if and only if there exists a finite sequence $\left(\boldsymbol{z}^{0}, \boldsymbol{z}^{1}, \ldots, \boldsymbol{z}^{h}\right)$ in $X$ such that $\boldsymbol{z}^{0}=\boldsymbol{x}, \boldsymbol{z}^{h}=\boldsymbol{y}$, and for every $k=1, \ldots, h$, there exist a pair of positive integers $i$ and $j$ and an $\epsilon>0$ such that

$$
z_{i}^{k}=z_{i}^{k-1}+\epsilon \leq z_{j}^{k-1}-\epsilon=z_{j}^{k} \text { and } z_{t}^{k-1}=z_{t}^{k} \text { for every } t \in \mathbb{N} \backslash\{i, j\} .
$$

\footnotetext{
${ }^{3}$ Observe that the assumptions of Theorem 1 and Theorem 2 are expressed in terms of $P(R)$, not $R$ itself, so that the indifference relation $I(R)$ has nothing to do with the validity of these propositions.
} 
It should be noted that $R_{\mathrm{PD}}$ is transitive. Note also that if a social evaluation relation $R$ satisfies $\mathbf{P D T}$ and $\boldsymbol{y} R_{\mathrm{PD}} \boldsymbol{x}$, then there is a finite sequence in $X$ starting from $\boldsymbol{x}$ and arriving at $\boldsymbol{y}$ that is increasingly desirable with respect to $P(R)$.

The following lemma due to Sakai (2006) proves useful in establishing Theorems 1 and 2 .

Lemma 1 [Sakai (2006, Lemma 6)] There exist an $\boldsymbol{x} \in X$ and a sequence $\left(\boldsymbol{x}^{n}\right)_{n \in \mathbb{N}}$ in $X$ such that $\boldsymbol{x}^{1} R_{\mathrm{PD}} \boldsymbol{x}, \boldsymbol{x}^{n+1} R_{\mathrm{PD}} \boldsymbol{x}^{n}$ for every $n \in \mathbb{N}$, and $\boldsymbol{x}^{n} \rightarrow \boldsymbol{x}$ as $n \rightarrow \infty$ in the sup topology.

Proof of Theorem 1. We prove that if a social evaluation relation satisfies PDT and PUSCs, then it is not acyclic. Let $R$ be a social evaluation relation satisfying PDT and PUSCs. Let $\boldsymbol{x}$ and $\left(\boldsymbol{x}^{n}\right)_{n \in \mathbb{N}}$ be as in Lemma 1. Since $\boldsymbol{x}^{n+1} R_{\mathrm{PD}} \boldsymbol{x}^{n}$ for every $n$, PDT implies that for every $n \geq 2$, there exists a finite sequence starting from $\boldsymbol{x}^{1}$ and arriving at $\boldsymbol{x}^{n}$ that is increasingly desirable with respect to $P(R)$. Since $\boldsymbol{x}^{1} R_{\mathrm{PD}} \boldsymbol{x}$ and $\boldsymbol{x}^{n} \rightarrow \boldsymbol{x}$, PUSCs implies that $\boldsymbol{x}^{1} P(R) \boldsymbol{x}^{n}$ for every sufficiently large $n$. Hence, for every sufficiently large $n$, there exists a $P(R)$-cycle involving $\boldsymbol{x}^{1}, \ldots, \boldsymbol{x}^{n}$. Thus $R$ is not acyclic.

Proof of Theorem 2. We prove by contradiction. Suppose that $R$ is a social evaluation relation satisfying LD and PUSCs. Let $\boldsymbol{x}$ and $\left(\boldsymbol{x}^{n}\right)_{n \in \mathbb{N}}$ be as in Lemma 1. Since $\boldsymbol{x}^{1} R_{\mathrm{PD}} \boldsymbol{x}, \mathbf{L D}$ implies that $\boldsymbol{x}^{1} P(R) \boldsymbol{x}$. From $\boldsymbol{x}^{n} \rightarrow \boldsymbol{x}$ as $n \rightarrow \infty$, PUSCs implies that $\boldsymbol{x}^{1} P(R) \boldsymbol{x}^{n}$ holds for every sufficiently large $n$. On the other hand, since $\boldsymbol{x}^{n+1} R_{\mathrm{PD}} \boldsymbol{x}^{n}$ for every $n, \boldsymbol{x}^{n}$ Lorenz dominates $\boldsymbol{x}^{1}$ for every $n \geq 2$. Thus, LD implies that $\boldsymbol{x}^{1} P(R) \boldsymbol{x}^{n}$ must not hold for any $n \geq 2$. This is a contradiction.

Two remarks concerning Theorems 1 and 2, in conjunction with the results of Sakai (2006), are in order. First, by making use of Lemma 4 in Sakai (2006) and by following the similar proof methods for Theorems 1 and 2, we can show that (i) there exists no acyclic social evaluation relation $R$ satisfying $\mathbf{P D T}$ and PLSCs; and (ii) there exists no social evaluation relation $R$ satisfying $\mathbf{L D}$ and PLSCs. Second, Sakai (2006) shows that there is no quasi-transitive social evaluation relation $R$ satisfying PDT and either RUSCs or RLSCs. It would therefore be fair to say that the impossibility theorems are robust with respect to the choice of continuity axioms introduced in the last section.

To identify the scope of our impossibility theorems, especially when the Pareto principle is additionally imposed, let us now consider weaker variants of the Pigou-Dalton transfer principle and the Lorenz domination principle: ${ }^{4}$

Weak Pigou-Dalton Transfer Principle (WPDT)

For any $\boldsymbol{x}, \boldsymbol{y} \in X$, and any pair of positive integers $i, j \in \mathbb{N}$, if there exists an $\epsilon>0$ such that (6) holds, then not $\boldsymbol{x} P(R) \boldsymbol{y}$.

Weak Lorenz Domination Principle (WLD)

\footnotetext{
${ }^{4}$ We thank an anonymous referee for suggesting this line of investigation.
} 
For any $\boldsymbol{x}, \boldsymbol{y} \in X$, if there exists $n^{*} \in \mathbb{N}$ such that (a) ${ }_{1} \boldsymbol{x}_{n^{*}}$ Lorenz dominates ${ }_{1} \boldsymbol{y}_{n^{*}}$, and (b) $x_{n}=y_{n}$ for all $n \in \mathbb{N}$ such that $n>n^{*}$, then not $\boldsymbol{y} P(R) \boldsymbol{x}$.

Since $P(R)$ is an asymmetric relation, these variants are indeed weaker than their original forms.

The first thing we should note on these variants is that they are so weak that there exist social evaluation relations satisfying both of them. As an example, take the social evaluation ordering $R$ that exhibits total indifference, that is, $\boldsymbol{x} R \boldsymbol{y}$ for all $\boldsymbol{x}, \boldsymbol{y} \in X$. Then, for any $\boldsymbol{x}, \boldsymbol{y} \in X, \boldsymbol{x} P(R) \boldsymbol{y}$ must not hold. Hence both WPDT and WLD are vacuously satisfied. All of the continuity assumptions introduced in the last section are also satisfied.

We are therefore led to consider whether these weaker variants are compatible with the Pareto principle. Here the issue of the possibility-impossibility divide becomes quite subtle, and we show that one version of the Pareto principle is compatible with both WPDT and WLD, but another is not, although the two versions would be equivalent were there to be only finitely many generations.

Specifically, we take up the following weak versions of the Pareto principle.

Weak Pareto Principle (WP)

For all $\boldsymbol{x}, \boldsymbol{y} \in X$, if $\boldsymbol{x} \gg \boldsymbol{y}$, then $\boldsymbol{x} P(R) \boldsymbol{y}$.

Very Weak Pareto Principle (VWP)

For all $\boldsymbol{x}, \boldsymbol{y} \in X$, if there exists an $\epsilon>0$ such that $\boldsymbol{x} \gg \boldsymbol{y}+\epsilon \mathbf{1}$, then $\boldsymbol{x} P(R) \boldsymbol{y}$.

VWP is weaker than WP. Yet VWP implies local non-satiation, viz., for every $\boldsymbol{y} \in X$ and every $\epsilon>0$, there exists an $\boldsymbol{x} \in X$ such that $d_{s}(\boldsymbol{x}, \boldsymbol{y})<\epsilon$ and $\boldsymbol{x} P(R) \boldsymbol{y}$. The following proposition shows that WPDT and WLD are compatible with VWP, all of the continuity assumptions, and some vestiges of the properties of an ordering.

\section{Proposition 1}

Define a social evaluation relation $R^{*}$ by

$$
\boldsymbol{x} R^{*} \boldsymbol{y} \text { if and only if } \inf _{n}\left(y_{n}-x_{n}\right) \leq 0
$$

for all $\boldsymbol{x}, \boldsymbol{y} \in X$. Then $R^{*}$ is complete, quasi-transitive (but not transitive), and satisfies WPDT, WLD, VWP, PUSCs, PLSCs, RUSCs, and RLSCs. ${ }^{5}, 6$

Proof of Proposition 1 We shall first prove that, for all $\boldsymbol{x}, \boldsymbol{y} \in X, \boldsymbol{x} P\left(R^{*}\right) \boldsymbol{y}$ if and only if there exists an $\epsilon>0$ such that $\boldsymbol{x} \gg \boldsymbol{y}+\epsilon \mathbf{1}$. In fact, $\boldsymbol{x} P\left(R^{*}\right) \boldsymbol{y}$ if and only if $\boldsymbol{x} R^{*} \boldsymbol{y}$ and not $\boldsymbol{y} R^{*} \boldsymbol{x}$, which is equivalent to saying that inf ${ }_{n}\left(y_{n}-x_{n}\right) \leq 0$ and $\inf _{n}\left(x_{n}-y_{n}\right)>0$. But the second inequality is equivalent to saying that

\footnotetext{
${ }^{5}$ As for the continuity properties, more can be said: $R^{*}$ is a closed subset of $X \times X$ and $P\left(R^{*}\right)$ is an open subset of $X \times X$ with respect to the product topology induced from the sup topology on $X$.

${ }^{6}$ The social evaluation relation $R^{*}$ satisfies the equity principle à la Sidgwick in the form of anonymity as well.
} 
$\boldsymbol{x} \gg \boldsymbol{y}+\epsilon \mathbf{1}$ for some $\epsilon>0$, which then implies that the first inequality must then be satisfied. Thus, $\boldsymbol{x} P\left(R^{*}\right) \boldsymbol{y}$ if and only if $\boldsymbol{x} \gg \boldsymbol{y}+\epsilon \mathbf{1}$ for some $\epsilon>0$. Hence $R^{*}$ satisfies VWP. It also follows from this fact that $R^{*}$ satisfies quasitransitivity, PUSCs, and PLSCs. In the above argument, we saw that if $\inf _{n}\left(x_{n}-y_{n}\right)>0$, then $\inf _{n}\left(y_{n}-x_{n}\right) \leq 0$. This shows that $R^{*}$ is complete. Hence $R^{*}$ also satisfies RUSCs and RLSCs. For all $\boldsymbol{x}, \boldsymbol{y} \in X$, if there exists an $n^{*} \in \mathbb{N}$ such that $x_{n}=y_{n}$ for every $n>n^{*}$, then we must not have $\boldsymbol{x} P\left(R^{*}\right) \boldsymbol{y}$. Thus $R^{*}$ satisfies WLD, and hence WPDT. Finally, to show that $R^{*}$ is not transitive, let $\boldsymbol{x}=\mathbf{1}, \boldsymbol{y}=\mathbf{1}+(0,1,0, \ldots)$ and $\boldsymbol{z}=2 \mathbf{1}$, where $\mathbf{1}$ is the infinite stream which repeats 1 indefinitely. Then, $\boldsymbol{x} R^{*} \boldsymbol{y}$ and $\boldsymbol{y} R^{*} \boldsymbol{z}$, as $\inf _{n}\left(y_{n}-x_{n}\right)=0$ and $\inf _{n}\left(z_{n}-y_{n}\right)=0$. But not $\boldsymbol{x} R^{*} \boldsymbol{z}$, as $\inf _{n}\left(z_{n}-x_{n}\right)=1$.

The next proposition shows that WPDT and WLD are incompatible with WP and PUSCs.

\section{Proposition 2}

(1) There exists no negatively transitive social evaluation relation satisfying WPDT, PUSCs and WP.

(2) There exists no social evaluation relation satisfying WLD, PUSCs and WP.

Proof of Proposition 2 (1) Suppose there were such a relation $R$. Let $\boldsymbol{x}=$ $\left(1,1 / 2,1 / 4,1 / 8, \cdots, 1 / 2^{n-1}, \cdots\right)$, and $\boldsymbol{x}^{n}=\left(s_{n} / n, s_{n} / n, \cdots, s_{n} / n, 1 / 2^{n}, \cdots\right)$, where $s_{n}=2\left(1-1 / 2^{n}\right)$ and there are $n$ terms of $s_{n} / n$ in $\boldsymbol{x}^{n}$. Note that $\boldsymbol{x}^{n+1} R_{\mathrm{PD}} \boldsymbol{x}^{n}$ for every $n$, because $R_{\mathrm{PD}}$ is transitive and $\boldsymbol{x}^{n+1}$ can be obtained from $\boldsymbol{x}^{n}$ by transferring $s_{n} / n-s_{n+1} /(n+1)$ from each of the first $n$ generations to the $(n+1)$-th generation. Then, since $R_{\mathrm{PD}}$ is transitive and $\boldsymbol{x}^{1}=\boldsymbol{x}$, $\boldsymbol{x}^{n} R_{\mathrm{PD}} \boldsymbol{x}$ for every $n$. By WPDT and the negative transitivity of $R$, we must have not $\boldsymbol{x} P(R) \boldsymbol{x}^{n}$ for each $n$. On the other hand, by WP, $\boldsymbol{x} P(R) \mathbf{0}$ implies that $\boldsymbol{x} P(R) \boldsymbol{x}^{n}$ for every large enough $n$ since $\boldsymbol{x}^{n} \rightarrow \mathbf{0}$ as $n \rightarrow \infty$, which is a contradiction.

(2) Suppose there were such a relation $R$. Consider $\boldsymbol{x}$ and $\boldsymbol{x}^{n}$ defined above. WP implies that $\boldsymbol{x} P(R) \mathbf{0}$. By PUSCs and noting that $\boldsymbol{x}^{n} \rightarrow \mathbf{0}$ as $n \rightarrow \infty$, we must have $\boldsymbol{x} P(R) \boldsymbol{x}^{n}$ for sufficiently large $n$. On the other hand, by WLD, not $\boldsymbol{x} P(R) \boldsymbol{x}^{n}$ for every $n$. This is a contradiction.

We may note in the proofs that, for our main impossibility results, a crucial step in the proofs is to establish the existence of $\boldsymbol{x}, \boldsymbol{y} \in X$ and a sequence $\left(\boldsymbol{x}^{n}\right)_{n \in \mathbb{N}}$ such that $\boldsymbol{x}^{n} \rightarrow \boldsymbol{x}, \boldsymbol{x}^{n} R_{P D} \boldsymbol{y}$, and $\boldsymbol{y} P(R) \boldsymbol{x}$. This may be established either by PDT and Lemma 1, or by LD and Lemma 1, or by WPDT and WP, or by WLD and WP.

\section{Robustness}

The impossibility theorems obtained so far commonly invoked the upper or lower semi-continuity of a social evaluation relation with respect to the sup topology. Yet, the foregoing studies on the evaluation of infinite utility streams, e.g. Campbell (1985), Lauwers (1997), Shinotsuka (1998) and Svensson (1980), 
showed that some impossibility results hinge squarely on the choice of a topology. The choice of a topology, with respect to which the semi-continuity requirement is given, matters to the validity of these impossibility theorems.

In this section, we show that our impossibility theorems are valid for a wide class of topologies. This result can be interpreted as justifying the extensive use of the sup topology in the literature. Our approach, however, can be distinguished from most of the foregoing studies in that we do not specify any particular topology other than the canonical sup topology to establish impossibility theorems. Rather, we consider classes of topologies satisfying certain conditions, such as linearity and local solidness, and clarify the relationship between the semi-continuity requirement with respect to these topologies, on the one hand, and the semi-continuity requirement with respect to the sup topology, on the other hand, to extend the impossibility theorems in the previous sections.

Let us start with locally solid linear topologies. For each $\boldsymbol{x} \in X$, let $|\boldsymbol{x}|$ be the infinite sequence of non-negative real numbers obtained from $\boldsymbol{x}$ by replacing each term $x_{n}$ with the absolute value thereof. A subset $A$ of $X$ is said to be solid if, for every $\boldsymbol{x}, \boldsymbol{y} \in l^{\infty},|\boldsymbol{x}|<|\boldsymbol{y}|$ and $\boldsymbol{y} \in A$ imply $\boldsymbol{x} \in A$. A linear topology on $l^{\infty}$ is said to be locally solid if it has a basis for $\mathbf{0}$ consisting of solid sets. The sup topology is locally solid, because the sets of the form $\left\{\boldsymbol{x} \in l^{\infty} \mid d_{s}(\boldsymbol{x}, \boldsymbol{y}) \leq \delta\right\}$ with $\delta>0$ define a fundamental system of neighborhoods of $\boldsymbol{y}$. In fact, it is easy to show that any other locally solid linear topology on $l^{\infty}$ is coarser than the sup topology. Since the semi-continuity requirement with respect to a coarser topology is a stronger restriction on social evaluation relations, all the impossibility theorems in Section 3 still hold when the semi-continuity with respect to the sup topology is replaced by the semi-continuity with respect to any other locally solid linear topology.

Next, we turn to a continuity requirement involving scalar multiplications.

Linear Upper Semi-Continuity (LUSC)

For all $\boldsymbol{x}, \boldsymbol{y} \in X,\left\{\alpha \in \mathbb{R} \mid \boldsymbol{y}+\alpha \mathbf{1} \in L_{P(R)}(\boldsymbol{x})\right\}$ is an open subset of $\{\alpha \in \mathbb{R} \mid \boldsymbol{y}+\alpha \mathbf{1} \in X\}$ (with respect to the Euclidean topology)

LUSC is nothing but the $\mathrm{P}$-upper semi-continuity when the comparison to a utility stream $\boldsymbol{x}$ is restricted to the line in the direction of $\mathbf{1}$ going through another utility stream $\boldsymbol{y}$. Similar conditions appeared in Herstein and Milnor (1953) in the context of expected utilities, and in Inoue (2006) in the context of equilibrium analysis.

\section{Lemma 2}

Every quasi-transitive social evaluation relation satisfying VWP and LUSC also satisfies PUSCs.

\section{Proof of Lemma $2^{7}$}

\footnotetext{
${ }^{7} \mathrm{~A}$ similar proof appeared in Inoue (2006).
} 
Let $R$ be a quasi-transitive social evaluation relation satisfying VWP and LUSC. Let $\boldsymbol{x} P(R) \boldsymbol{y}$. It suffices to show that there exists a neighborhood $Y$ of $\boldsymbol{y}$ in $X$ with respect to the sup topology such that $\boldsymbol{x} P(R) \boldsymbol{z}$ for every $\boldsymbol{z} \in Y$.

Define $A=\{\alpha \in \mathbb{R} \mid \boldsymbol{y}+\alpha \mathbf{1} \in X\}$ and $B=\left\{\alpha \in \mathbb{R} \mid \boldsymbol{y}+\alpha \mathbf{1} \in L_{P(R)}(\boldsymbol{x})\right\}$, then $A \supseteq \mathbb{R}_{+}, B \ni 0$, and, by LUSC, $B$ is an open subset of $A$. Hence there exists a $\delta>0$ such that $\delta \in B$, that is,

$$
\boldsymbol{x} P(R)(\boldsymbol{y}+\delta \mathbf{1}) .
$$

Define $Y=\{\boldsymbol{z} \in X \mid$ there exists an $\alpha<\delta$ such that $\boldsymbol{y}+\alpha \mathbf{1} \gg \boldsymbol{z}\}$. Then $\boldsymbol{y} \in Y$. We now prove that $Y$ is an open subset of $X$. Let $\boldsymbol{z} \in Y$. Then there exists an $\alpha<\delta$ such that $\boldsymbol{y}+\alpha \mathbf{1} \gg \boldsymbol{z}$. Then, for every $\boldsymbol{w} \in X$, if $d_{s}(\boldsymbol{w}, \boldsymbol{z})<\delta-\alpha$, then

$$
\boldsymbol{w} \ll \boldsymbol{z}+d_{s}(\boldsymbol{w}, \boldsymbol{z}) \mathbf{1} \ll(\boldsymbol{y}+\alpha \mathbf{1})+d_{s}(\boldsymbol{w}, \boldsymbol{z}) \mathbf{1}=\boldsymbol{y}+\left(\alpha+d_{s}(\boldsymbol{w}, \boldsymbol{z})\right) \mathbf{1} .
$$

Since $\alpha+d_{s}(\boldsymbol{w}, \boldsymbol{z})<\delta$, this show that $\boldsymbol{w} \in Y$. Hence $Y$ is an open subset of $X$.

For every $\boldsymbol{z} \in Y$, there exists an $\epsilon>0$ such that $\boldsymbol{y}+\delta \mathbf{1} \gg \boldsymbol{z}+\epsilon \mathbf{1}$. Hence, by VWP,

$$
(\boldsymbol{y}+\delta \mathbf{1}) P(R) \boldsymbol{z}
$$

By (4), (5), and quasi-transitivity, $\boldsymbol{x} P(R) \boldsymbol{z}$.

In place of LUSC, we could use an alternative version of linear upper semicontinuity, which requires $\left\{\alpha \in \mathbb{R} \mid \boldsymbol{y}+\alpha \mathbf{1} \in U_{R}(\boldsymbol{x})\right\}$ to be a closed subset of $\{\alpha \in \mathbb{R} \mid \boldsymbol{y}+\alpha \mathbf{1} \in X\}$ for all $\boldsymbol{x}, \boldsymbol{y} \in X$. We could then show that every transitive social evaluation relation satisfying $\mathbf{V W P}$ and this version of linear upper semi-continuity also satisfies RUSCs. If $R$ is complete, then the two versions of linear upper semi-continuity are equivalent. Thus, every social evaluation ordering satisfying VWP and either of the two upper semi-continuity axioms also satisfies PUSCs and RUSCs.

It immediately follows from the impossibility theorems in Section 3 and Lemma 2 that there is no quasi-transitive social evaluation relation which satisfies VWP, LUSC, either PDT or LD.

We now explore two further implications of LUSC. Note first that since the scalar multiplication $\alpha \mapsto \boldsymbol{y}+\alpha \mathbf{1}$ is continuous with respect to every linear topology on $l^{\infty}$, if the upper semi-continuity is satisfied for some linear topology, then LUSC is satisfied as well. Thus, for quasi-transitive social evaluation relations satisfying VWP, the impossibility theorems in Section 3 can be generalized to the case in which $L_{P(R)}(\boldsymbol{x})$ is open with respect to an arbitrary linear topology. This generalization is not vacuous. Indeed, Kolmogorov and Fomin (1971, Problem 10 of Section 17, Chapter 4) describe how to construct a linear topology stronger than the sup topology.

The second implication of LUSC is concerned with social evaluation orderings that can be represented by concave utility functions. We define $X^{*}$ as the set of all $\boldsymbol{x} \in X$ for which there exists a $\delta>0$ such that $\boldsymbol{x}-\delta \mathbf{1} \in X$. We then claim that there exists no social evaluation ordering $R$ on the domain $X$ which is represented by a concave function $f: X \rightarrow \mathbb{R}$, and the restriction of 
which onto $X^{*}$ satisfies VWP and either PDT or LD. This claim can easily be proved based on the fact that every concave function defined on an interval of the real line is continuous on the interior of the interval.

To appreciate the significance of this extension, recall that a Bergson-Samuelson social welfare function is a function that maps each possible profile of utility levels for finitely many individuals to a single social utility level. In the welfare analysis, it is common to assume that a Bergson-Samuelson social welfare function satisfies the Pareto Principle to embody the idea of respecting efficiency, as well as concavity to embody the idea of respecting egalitarianism. The function $f: X \rightarrow \mathbb{R}$ can be considered as an extension of a Bergson-Samuelson social welfare function to the case of infinitely many individuals. Our result then shows that once the Pareto principle and concavity are accepted for an infinite-dimensional Bergson-Samuelson social welfare function, the upper semicontinuity with respect to the sup topology is no additional restriction on social welfare functions as long as the comparison is restricted onto the utility streams that are uniformly bounded away from the minimum levels in $X$.

\section{Concluding Remarks}

In view of the recent plethora of possibility/impossibility theorems on the evaluation of infinite utility streams, it is of necessity that we have a scheme of logical classifications of relevant results. There are three criteria that play crucial role in what follows.

The first criterion is about the rationality condition that is required of the social evaluation relation, viz., completeness, transitivity, negative transitivity, acyclicity, and the like. The second criterion is the robustness of the evaluation ranking to a small perturbation of infinite utility streams, viz., whether or not the social evaluation relation satisfies continuity, upper semi-continuity, and lower semi-continuity with respect to some appropriate topology on the domain. The third criterion is about the sensitivity conditions imposed on the social evaluation relation, and we make use of the two types of conditions in our scheme. The first type is the sensitivity to efficiency, viz., whether or not it satisfies the Pareto principle, the weak Pareto principle, and the strong Pareto principle. The second type is the sensitivity to equity, viz., whether or not it satisfies the procedural fairness in the sense of the Aristotle-Sidgwick anonymity, the distributional egalitarianism such as the Pigou-Dalton transfer principle or the Lorenz domination principle, and the like.

Invoking this classificatory scheme, the classical Diamond impossibility theorem can be characterized by the combination of

D(1) completeness and transitivity,

$\mathbf{D}(2)$ continuity with respect to the sup topology, and

D(3) (i) strong Pareto and (ii) anonymity.

Theorems 1 and 2 of Sakai (2006) can be characterized by the combinations of $\mathbf{S}(1)$ quasi-transitivity, 
$\mathbf{S}(2)$ upper or lower semi-continuity with respect to the sup topology, and

S(3) (i) none and (ii) Pigou-Dalton transfer principle.

Likewise, our Theorem 1 can be characterized by the combination of

HSSX1(1) acyclicity,

HSSX1(2) upper or lower semi-continuity with respect to the sup topology,

HSSX1(3) (i) none and (ii) Pigou-Dalton transfer principle,

whereas our Theorem 2 can be characterized by the combination of

HSSX2(1) none,

HSSX2(2) upper or lower semi-continuity with respect to the sup topology

HSSX2(3) (i) none and (ii) Lorenz domination principle.

Needless to say, most if not all impossibility theorems in this arena can be similarly characterized. It is our hope that the classification of the impossibility theorems along this line will be found useful in bringing the nature and significance of our results to the fore.

In concluding this paper, a final remark may be in order. Although our analysis relied heavily on the semi-continuity requirement on social evaluation relation, Basu and Mitra (2003) did not impose any such requirement. Instead, they assumed that social evaluation relations must be representable by numerical functions. Representability implies completeness and transitivity. Hence any impossibility theorem that does not impose completeness or transitivity is outside the scope of their impossibility theorems. This highlights the difference between their and our approaches, as our impossibility theorems use acyclicity, or even less, in place of completeness and transitivity. The same can be said of the approach by Zame (2007). As he himself exemplified, one of his theorems, which asserts that the existence of a (complete and transitive) social evaluation ordering satisfying the Pareto principle and the Aristotle-Sidgwick equity principle hinges crucially on the axiom of choice, would not survive once the completeness were dispensed with. 


\section{References}

Arrow, K. J. (1951): Social Choice and Individual Values, New York: John Wiley \& Sons. Second Edition, 1963.

Atkinson, A. B. (1970): "On the Measurement of Inequality," Journal of Economic Theory 2, pp.244-263. Reprinted in Atkinson, A. B., Social Justice and Public Policy, Cambridge, Mass.: The MIT Press, 1983.

Asheim, G. B. and B. Tungodden (2004): "Resolving Distributional Conflicts between Generations," Economic Theory 24, pp.221-230.

Banerjee, K. and T. Mitra (2007a): "On the Impatience Implications of Paretian Social Welfare Functions," Journal of Mathematical Economics 43, pp.236-248.

Banerjee, K. and T. Mitra (2007b): "On the Continuity of Ethical Social Welfare Orders of Infinite Utility Streams," forthcoming in Social Choice and Welfare.

Basu, K. and T. Mitra (2003): "Aggregating Infinite Utility Streams with Intergenerational Equity: Impossibility of Being Paretian," Econometrica 71, pp.1557-1563.

Basu, K. and T. Mitra (2007): "Utilitarianism for Infinite Utility Streams: A New Welfare Criterion and Its Axiomatic Characterization," Journal of Economic Theory 133, pp.350-373.

Bossert, W., Y. Sprumont and K. Suzumura (2005): "Ordering Infinite Utility Streams," forthcoming in Journal of Economic Theory.

Campbell, D. E. (1985): "Impossibility Theorems and Infinite Horizon Planning," Social Choice and Welfare 2, pp.283-293.

Diamond, P. A. (1965): "The Evaluation of Infinite Utility Streams," Econometrica 33, pp.170-177.

Fleurbaey, M. and P. Michel (2001): "Transfer Principles and Inequality Aversion, with an Application to Optimal Growth," Mathematical Social Sciences 42, pp.1-11.

Fleurbaey, M. and P. Michel (2003): "Intertemporal Equity and the Extension of the Ramsey Criterion," Journal of Mathematical Economics 39, pp.777802.

Hammond, P. (1976): “Equity, Arrow's Conditions, and Rawls' Difference Principle," Econometrica 44, pp. 793-804.

Hara, C., T. Shinotsuka, K. Suzumura and Y. Xu (2005): "On the Possibility of Continuous, Paretian and Egalitarian Evaluation of Infinite Utility Streams," Working Paper, Institute of Economic Research, Hitotsubashi University. 
Herstein, I. N. and J. Milnor (1953): "An Axiomatic Approach to Measurable Utility," Econometrica 21, pp.291-297.

Inoue, T. (2006): "Linearly Continuous Preferences and Equilibrium Analysis in a Finite Dimensional Commodity Space," unpublished manuscript, Institute of Economic Research, Kyoto University.

Kolmogorov, A. N. and S. V. Fomin (1971): Introductory Real Analysis, translated and edited by R. A. Silverman, New York: Dover Publications, INC.

Koopmans, T. C. (1960): "Stationary Ordinal Utility and Impatience," Econometrica 28, pp.287-309.

Lauwers, L. (1997): "Continuity and Equity with Infinite Horizons," Social Choice and Welfare 14, pp.345-356.

Lauwers, L. (2007): "Ordering Infinite Utility Streams: Completeness at the Cost of a Non-Ramsey Set," K. U. Leuven, Unpublished Manuscript.

Mas-Colell, A. (1986): "The Price Equilibrium Existence Theorem in Topological Vector Lattices," Econometrica 54, pp.1039-1054.

Sakai, T. (2006): "Equitable Intergenerational Preferences on Restricted Domains," Social Choice and Welfare 27, pp.41-54.

Sen, A. K. (1970): Collective Choice and Social Welfare, San Francisco: HoldenDay. Republished, Amsterdam: North-Holland, 1979.

Sen, A. K. (1997): On Economic Inequality, Expanded edition with a substantial annexe by James E. Foster and Amartya Sen, Oxford: Clarendon Press.

Shinotsuka, T. (1998): "Equity, Continuity, and Myopia: A Generalization of Diamond's Impossibility Theorem," Social Choice and Welfare 15, pp.2130 .

Sidgwick, H. (1907): The Methods of Ethics, 7th edition, London: Macmillan and Co.

Svensson, L.-G. (1980): "Equity among Generations," Econometrica 48, pp.12511256.

Zame, W. R. (2007): "Can Intergenerational Equity be Operationalized?" Theoretical Economics 2, pp.187-202. 